Some sharp function estimates for vector-valued multilinear integral operator

\author{
Xiaosha Zhou
}




\title{
SOME SHARP FUNCTION ESTIMATES FOR VECTOR-VALUED MULTILINEAR INTEGRAL OPERATOR
}

\author{
XIAOSHA ZHOU \\ Received 10 October, 2012
}

\begin{abstract}
In this paper, we establish some sharp estimates for certain vector-valued multilinear integral operators. The operators include Littlewood-Paley operators, Marcinkiewicz operators and the Bochner-Riesz operator. As an application, we obtain the $\left(L^{p}, L^{q}\right)$-norm inequality for the vector-valued multilinear operators.
\end{abstract}

2000 Mathematics Subject Classification: 42B20; 42B25

Keywords: vector-valued multilinear operator, Littlewood-Paley operator, Marcinkiewicz operator, Bochner-Riesz operator, Sharp estimate, BMO

\section{INTRODUCTION}

As a part of the development of singular integral operators, their commutators and multilinear operators have been well studied in [2-5, 14-18]. In [8], Hu and Yang obtained a variant sharp estimate for the multilinear singular integral operators. In [18], C. Pérez and R. Trujillo-Gonzalez obtained a sharp weighted estimate for the vector-valued singular integral operators and their commutators. The main purpose of this paper is to prove the sharp estimates for some multilinear operators related to certain integral operators. The integral operators include Littlewood-Paley operator, Marcinkiewicz operator and the Bochner-Riesz operator. As an applications, we obtain the $\left(L^{p}, L^{q}\right)$-norm inequalities for the multilinear operators.

\section{NotATIONS AND THEOREMS}

Let $m_{j}(j=1, \cdots, l)$ be positive integers with $m_{1}+\cdots+m_{l}=m, A_{j}: R^{n} \rightarrow$ $\mathcal{C}(j=1, \cdots, l), F_{t}: R^{n} \times R^{n} \times[0,+\infty) \rightarrow \mathcal{C}$ and $(x, y, t) \mapsto F_{t}(x, y)$ be some locally integrable functions. Set

and

$$
F_{t}(f)(x):=\int_{R^{n}} F_{t}(x, y) f(y) d y
$$

$$
F_{t}^{A}(f)(x):=\int_{R^{n}} \frac{\prod_{j=1}^{l} R_{m_{j}+1}\left(A_{j} ; x, y\right)}{|x-y|^{m}} F_{t}(x, y) f(y) d y
$$


for every bounded and compactly supported function $f$, where

$$
R_{m_{j}+1}\left(A_{j} ; x, y\right)=A_{j}(x)-\sum_{|\alpha| \leq m_{j}} \frac{1}{\alpha !} D^{\alpha} A_{j}(y)(x-y)^{\alpha} .
$$

In addition $F_{t}$ satisfies the following condition: for fixed $\varepsilon>0$ and $0 \leq \delta<n$,

$$
\left\|F_{t}(x, y)\right\| \leq C|x-y|^{-n+\delta}
$$

and

$$
\left\|F_{t}(y, x)-F_{t}(z, x)\right\| \leq C|y-z|^{\varepsilon}|x-z|^{-n-\varepsilon+\delta}
$$

if $2|y-z| \leq|x-z|$. For $1<r<\infty$ and for the vector-valued multilinear operators $\left|T^{A}(f)\right|_{r}$ and $|T(f)|_{r}$ the bounded and compactly supported functions on $R^{n} \rightarrow$ $H$ related to $F_{t}^{A}$ are defined by

$\left|T^{A}(f)(x)\right|_{r}:=\left(\sum_{i=1}^{\infty}\left(T^{A}\left(f_{i}\right)(x)\right)^{r}\right)^{1 / r}$ and $|T(f)(x)|_{r}:=\left(\sum_{i=1}^{\infty}\left|T\left(f_{i}\right)(x)\right|^{r}\right)^{1 / r}$,

where

$$
T^{A}\left(f_{i}\right)(x):=\left\|F_{t}^{A}\left(f_{i}\right)(x)\right\|, \quad T\left(f_{i}\right)(x):=\left\|F_{t}\left(f_{i}\right)(x)\right\| .
$$

Here $\|\cdot\|$ is the norm of the Banach space $H:=$ \{continous and bounded $h: R^{n} \rightarrow \ell$ \} with norm $\|h\|$ such that, for each fixed $x \in R^{n}, F_{t}(f)(x)$ and $F_{t}^{A}(f)(x)$ may be viewed as a mapping from $[0,+\infty)$ to $H$. Set

$$
|f(x)|_{r}:=\left(\sum_{i=1}^{\infty}\left|f_{i}(x)\right|^{r}\right)^{1 / r} .
$$

Suppose that $|T|_{r}$ is bounded from $L^{p}\left(R^{n}\right)$ to $L^{q}\left(R^{n}\right)$ for any $1<p<n / \delta$ and $1 / q=1 / p-\delta / n$.

If $m=0, T^{A}$ is just the vector-valued multilinear commutator of $T$ and $A$ (see [15]). If $m>0, T^{A}$ is non-trivial generalizations of the commutator. It is well known that multilinear operators are of great interest in harmonic analysis and have been widely studied by many authors (see $[2-5,14]$ ). The main purpose of this paper is to prove a sharp inequality for the vector-valued multilinear integral operators $T^{A}$. As an application, we obtain a $\left(L^{p}, L^{q}\right)$-norm inequality for the vector-valued multilinear operators. In Section 4, we give some examples.

First, let us introduce some notations. Throughout this paper, $Q$ will denote a cube of $R^{n}$ with sides parallel to the axes. For any locally integrable function $f: R^{n} \rightarrow \mathcal{C}$, define $f_{Q}=|Q|^{-1} \int_{Q} f(x) d x$ and

$$
f^{\#}(x):=\sup _{Q \ni x} \frac{1}{|Q|} \int_{Q}\left|f(y)-f_{Q}\right| d y,
$$


and set $\|f\|_{B M O}:=\left\|f^{\#}\right\|_{L^{\infty}}$. We write $f \in B M O\left(R^{n}\right)$ if $\|f\|_{B M O}<\infty$. It is well-known that (see [7])

$$
f^{\#}(x) \approx \sup _{Q \ni x} \inf _{c \in C} \frac{1}{|Q|} \int_{Q}|f(y)-c| d y .
$$

For $1 \leq p<\infty$ and $0 \leq \delta<n$, let

$$
M_{\delta, p}(f)(x):=\sup _{Q \ni x}\left(\frac{1}{|Q|^{1-p \delta / n}} \int_{Q}|f(y)|^{p} d y\right)^{1 / p} .
$$

We shall prove the following theorems.

Theorem 1. If $1<r<\infty, D^{\alpha} A_{j} \in B M O\left(R^{n}\right)$ for all $\alpha$ with $|\alpha|=m_{j}(j=$ $1, \cdots, l)$, then there exists a constant $C>0$ such that for any $f=\left\{f_{i}\right\} \in C_{0}^{\infty}\left(R^{n}\right)$, $1<s<n / \delta$ and $\tilde{x} \in R^{n}$,

$$
\left(\left|T^{A}(f)\right|_{r}\right)^{\#}(\tilde{x}) \leq C \prod_{j=1}^{l}\left(\sum_{\left|\alpha_{j}\right|=m_{j}}\left\|D^{\alpha_{j}} A_{j}\right\|_{B M O}\right) M_{\delta, s}\left(|f|_{r}\right)(\tilde{x}) .
$$

Theorem 2. If $D^{\alpha} A_{j} \in B M O\left(R^{n}\right)$ for all $\alpha$ with $|\alpha|=m_{j}(j=1, \cdots, l)$, then $\left|T^{A}\right|_{r}: L^{p}\left(R^{n}\right) \rightarrow L^{q}\left(R^{n}\right)$ is bounded for $1<r<\infty, 1<p<n / \delta$ and $1 / p-1 / q=$ $\delta / n$, that is

$$
\left\|\left|T^{A}(f)\right|_{r}\right\|_{L^{q}} \leq C \prod_{j=1}^{l}\left(\sum_{\left|\alpha_{j}\right|=m_{j}}\left\|D^{\alpha_{j}} A_{j}\right\|_{B M O}\right)\left\|\left|\left\|\left.f\right|_{r}\right\|_{L^{p}} .\right.\right.
$$

\section{Proofs of the Theorems}

To prove the theorems, we need the following lemmas.

Lemma 1 (see [4]). Let $A: R^{n} \rightarrow \mathcal{C}$ be a locally integrable function and $D^{\alpha} A \in$ $L^{q}\left(R^{n}\right)$ for all $\alpha$ with $|\alpha|=m$ and some $q>n$. Then

$$
\left|R_{m}(A ; x, y)\right| \leq C|x-y|^{m} \sum_{|\alpha|=m}\left(\frac{1}{|\tilde{Q}(x, y)|} \int_{\tilde{Q}(x, y)}\left|D^{\alpha} A(z)\right|^{q} d z\right)^{1 / q},
$$

where $\tilde{Q}$ is the cube centered at $x$ and having side length $5 \sqrt{n}|x-y|$.

Lemma 2 (see [1,6]). Suppose that $1<r<\infty, 0 \leq \delta<n, 1 \leq s<p<n / \delta$ and $1 / q=1 / p-\delta / n$. Then

$$
\left\|M_{\delta, s}\left(|f|_{r}\right)\right\|_{L^{q}} \leq C\left|\left\|\left.f\right|_{r}\right\|_{L^{p}} .\right.
$$


Proof of Theorem 1. It suffices to prove for $f=\left\{f_{i}\right\} \in C_{0}^{\infty}\left(R^{n}\right)$ and some cons$\operatorname{tant} C_{0}$, the following inequality:

$$
\frac{1}{|Q|} \int_{Q} \|\left. T^{A}(f)(x)\right|_{r}-C_{0} \mid d x \leq C \prod_{j=1}^{l}\left(\sum_{\left|\alpha_{j}\right|=m_{j}}\left\|D^{\alpha_{j}} A_{j}\right\|_{B M O}\right) M_{\delta, s}(|f| r)(\tilde{x}) .
$$

Without loss of generality, we may assume $l=2$. Fix a cube $Q=Q\left(x_{0}, d\right)$ and $\tilde{x} \in$ $Q$. Let $\tilde{Q}=5 \sqrt{n} Q$ and $\tilde{A}_{j}(x)=A_{j}(x)-\sum_{|\alpha|=m_{j}} \frac{1}{\alpha !}\left(D^{\alpha} A_{j}\right) \tilde{Q}^{x^{\alpha}}$, then $R_{m_{j}}\left(A_{j} ; x, y\right)$ $=R_{m_{j}}\left(\tilde{A}_{j} ; x, y\right)$ and $D^{\alpha} \tilde{A}_{j}=D^{\alpha} A_{j}-\left(D^{\alpha} A_{j}\right)_{\tilde{Q}}$ for $|\alpha|=m_{j}$. We split $f=$ $g+h=\left\{g_{i}\right\}+\left\{h_{i}\right\}$ for $g_{i}=f_{i} \chi_{\tilde{Q}}$ and $h_{i}=f_{i} \chi_{R^{n} \backslash \tilde{Q}}$. Write

$$
\begin{aligned}
& F_{t}^{A}\left(f_{i}\right)(x)=\int_{R^{n}} \frac{\prod_{j=1}^{2} R_{m_{j}+1}\left(\tilde{A}_{j} ; x, y\right)}{|x-y|^{m}} F_{t}(x, y) f_{i}(y) d y \\
& =\int_{R^{n}} \frac{\prod_{j=1}^{2} R_{m_{j}+1}\left(\tilde{A}_{j} ; x, y\right)}{|x-y|^{m}} F_{t}(x, y) h_{i}(y) d y \\
& +\int_{R^{n}} \frac{\prod_{j=1}^{2} R_{m_{j}}\left(\tilde{A}_{j} ; x, y\right)}{|x-y|^{m}} F_{t}(x, y) g_{i}(y) d y \\
& -\sum_{\left|\alpha_{1}\right|=m_{1}} \frac{1}{\alpha_{1} !} \int_{R^{n}} \frac{R_{m_{2}}\left(\tilde{A}_{2} ; x, y\right)(x-y)^{\alpha_{1}}}{|x-y|^{m}} D^{\alpha_{1}} \tilde{A}_{1}(y) F_{t}(x, y) g_{i}(y) d y \\
& -\sum_{\left|\alpha_{2}\right|=m_{2}} \frac{1}{\alpha_{2} !} \int_{R^{n}} \frac{R_{m_{1}}\left(\tilde{A}_{1} ; x, y\right)(x-y)^{\alpha_{2}}}{|x-y|^{m}} D^{\alpha_{2}} \tilde{A}_{2}(y) F_{t}(x, y) g_{i}(y) d y \\
& +\sum_{\left|\alpha_{1}\right|=m_{1},\left|\alpha_{2}\right|=m_{2}} \frac{1}{\alpha_{1} ! \alpha_{2} !} \int_{R^{n}} \frac{(x-y)^{\alpha_{1}+\alpha_{2}} D^{\alpha_{1}} \tilde{A}_{1}(y) D^{\alpha_{2}} \tilde{A}_{2}(y)}{|x-y|^{m}} F_{t}(x, y) g_{i}(y) d y,
\end{aligned}
$$

then, by Minkowski' inequality,

$$
\begin{gathered}
\left.\frac{1}{|Q|} \int_{Q}|| T^{A}(f)(x)\right|_{r}-\left|T^{\tilde{A}}(h)\left(x_{0}\right)\right|_{r} \mid d x \\
\leq \frac{1}{|Q|} \int_{Q}\left|\left\|F_{t}^{A}(f)(x)\right\|_{r}-\left\|F_{t}^{\tilde{A}}(h)\left(x_{0}\right)\right\|_{r}\right| d x \\
\leq \frac{1}{|Q|} \int_{Q}\left(\sum_{i=1}^{\infty} \| F_{t}^{A}\left(f_{i}\right)(x)-\left.F_{t}^{\tilde{A}}\left(h_{i}\right)\left(x_{0}\right)\right|^{r}\right)^{1 / r} d x \\
\leq \frac{1}{|Q|} \int_{Q}\left(\sum_{i=1}^{\infty}\left\|\int_{R^{n}} \frac{\prod_{j=1}^{2} R_{m_{j}}\left(\tilde{A}_{j} ; x, y\right)}{|x-y|^{m}} F_{t}(x, y) g_{i}(y) d y\right\|^{r}\right)^{1 / r} d x
\end{gathered}
$$




$$
\begin{aligned}
& +\frac{C}{|Q|} \int_{Q}\left(\sum_{i=1}^{\infty} \| \sum_{\left|\alpha_{1}\right|=m_{1}}\right. \\
& \left.\qquad \int_{R^{n}} \frac{R_{m_{2}}\left(\tilde{A}_{2} ; x, y\right)(x-y)^{\alpha_{1}}}{|x-y|^{m}} D^{\alpha_{1}} \tilde{A}_{1}(y) F_{t}(x, y) g_{i}(y) d y \|^{r}\right)^{1 / r} d x \\
& +\frac{C}{|Q|} \int_{Q}\left(\sum_{i=1}^{\infty} \| \sum_{\left|\alpha_{2}\right|=m_{2}}\right. \\
& +\frac{C}{|Q|} \int_{Q}\left(\sum_{i=1}^{\infty}\left\|{ }_{R^{n}} \frac{R_{m_{1}}\left(\tilde{A}_{1} ; x, y\right)(x-y)^{\alpha_{2}}}{|x-y|^{m}} D^{\alpha_{2}} \tilde{A}_{2}(y) F_{t}(x, y) g_{i}(y) d y\right\|^{r}\right)^{1 / r} d x \\
& +\frac{1}{|Q|} \int_{Q}\left(\sum_{i=1}^{\infty} \| F_{1},\left|\alpha_{2}\right|=m_{2}\right. \\
& :=I_{1}+I_{2}+I_{3}+I_{4}+I_{5} .
\end{aligned}
$$

Now, let us estimate $I_{1}, I_{2}, I_{3}, I_{4}$ and $I_{5}$, respectively. First, for $x \in Q$ and $y \in \tilde{Q}$, we get by Lemma 1 ,

$$
R_{m}\left(\tilde{A}_{j} ; x, y\right) \leq C|x-y|^{m} \sum_{\left|\alpha_{j}\right|=m}\left\|D^{\alpha_{j}} A_{j}\right\|_{B M O},
$$

thus, by the $\left(L^{s}, L^{q}\right)$-boundedness of $|T|_{r}$ with $1<s<n / \delta$ and $1 / q=1 / s-\delta / n$, we obtain

$$
\begin{aligned}
I_{1} & \leq C \prod_{j=1}^{2}\left(\sum_{\left|\alpha_{j}\right|=m_{j}}\left\|D^{\alpha_{j}} A_{j}\right\|_{B M O}\right) \frac{1}{|Q|} \int_{Q}|T(g)(x)|_{r} d x \\
& \leq C \prod_{j=1}^{2}\left(\sum_{\left|\alpha_{j}\right|=m_{j}}\left\|D^{\alpha_{j}} A_{j}\right\|_{B M O}\right)\left(\frac{1}{|Q|} \int_{Q}|T(g)(x)|_{r}^{q} d x\right)^{1 / q} \\
& \leq C \prod_{j=1}^{2}\left(\sum_{\left|\alpha_{j}\right|=m_{j}}\left\|D^{\alpha_{j}} A_{j}\right\|_{B M O}\right)|Q|^{-1 / q}\left(\int_{\tilde{Q}}|f(x)|_{r}^{S} d x\right)^{1 / s}
\end{aligned}
$$




$$
\leq C \prod_{j=1}^{2}\left(\sum_{\left|\alpha_{j}\right|=m_{j}}\left\|D^{\alpha_{j}} A_{j}\right\|_{B M O}\right) M_{\delta, s}\left(|f|_{r}\right)(\tilde{x}) .
$$

For $I_{2}$, denoting $s=p q$ for $1<p<n / \delta, q>1,1 / q+1 / q^{\prime}=1$ and $1 / u=1 / p-$ $\delta / n$, we get, by Hölder's inequality,

$$
\begin{aligned}
I_{2} & \leq C \sum_{\left|\alpha_{2}\right|=m_{2}}\left\|D^{\alpha_{2}} A_{2}\right\|_{B M O} \sum_{\left|\alpha_{1}\right|=m_{1}} \frac{1}{|Q|} \int_{Q}\left|T\left(D^{\alpha_{1}} \tilde{A}_{1} g\right)(x)\right|_{r} d x \\
& \leq C \sum_{\left|\alpha_{2}\right|=m_{2}}|| D^{\alpha_{2}} A_{2} \|_{B M O} \sum_{\left|\alpha_{1}\right|=m_{1}}\left(\frac{1}{|Q|} \int_{R^{n}}\left|T\left(D^{\alpha_{1}} \tilde{A}_{1} g\right)(x)\right|_{r}^{u} d x\right)^{1 / u} \\
& \leq C \sum_{\left|\alpha_{2}\right|=m_{2}}|| D^{\alpha_{2}} A_{2} \|_{B M O} \sum_{\left|\alpha_{1}\right|=m_{1}}|Q|^{-1 / u}\left(\int_{R^{n}}\left(\left|D^{\alpha_{1}} \tilde{A}_{1}(x)\right||g(x)|_{r}\right)^{p} d x\right)^{1 / p} \\
& \leq C \sum_{\left|\alpha_{2}\right|=m_{2}}|| D^{\alpha_{2}} A_{2} \|_{B M O} \sum_{\left|\alpha_{1}\right|=m_{1}}\left(\frac{1}{|Q|} \int_{\tilde{Q}}\left|D^{\alpha_{1}} \tilde{A}_{1}(x)\right|^{p q^{\prime}} d x\right)^{1 / p q^{\prime}} \\
& \times\left(\frac{1}{|Q|^{1-s \delta / n}} \int_{\tilde{Q}}|f(x)|_{r}^{p q} d x\right)^{1 / p q} \\
& \leq C \prod_{j=1}^{2}\left(\sum_{|\alpha|=m_{j}}|| D^{\alpha} A_{j} \|_{B M O}\right) M_{\delta, s}\left(|f|_{r}\right)(\tilde{x}) .
\end{aligned}
$$

For $I_{3}$, similar to the proof of $I_{2}$, we get

$$
I_{3} \leq C \prod_{j=1}^{2}\left(\sum_{|\alpha|=m_{j}}\left\|D^{\alpha} A_{j}\right\|_{B M O}\right) M_{\delta, s}\left(|f|_{r}\right)(\tilde{x}) .
$$

Similarly, for $I_{4}$, denoting $s=p q_{3}$ for $1<p<n / \delta, q_{1}, q_{2}, q_{3}>1,1 / q_{1}+1 / q_{2}+$ $1 / q_{3}=1$ and $1 / u=1 / p-\delta / n$, we obtain

$$
\begin{aligned}
I_{4} \leq C \sum_{\left|\alpha_{1}\right|=m_{1},\left|\alpha_{2}\right|=m_{2}} \frac{1}{|Q|} \int_{Q}\left|T\left(D^{\alpha_{1}} \tilde{A}_{1} D^{\alpha_{2}} \tilde{A}_{2} g\right)(x)\right|_{r} d x \\
\leq C \sum_{\left|\alpha_{1}\right|=m_{1},\left|\alpha_{2}\right|=m_{2}}\left(\frac{1}{|Q|} \int_{R^{n}}\left|T\left(D^{\alpha_{1}} \tilde{A}_{1} D^{\alpha_{2}} \tilde{A}_{2} g\right)(x)\right|_{r}^{u} d x\right)^{1 / u} \\
\leq C \sum_{\left|\alpha_{1}\right|=m_{1},\left|\alpha_{2}\right|=m_{2}}|Q|^{-1 / t}\left(\int_{R^{n}}\left(\left|D^{\alpha_{1}} \tilde{A}_{1}(x) D^{\alpha_{2}} \tilde{A}_{2}(x)\right||g(x)|_{r}\right)^{p} d x\right)^{1 / p}
\end{aligned}
$$




$$
\begin{gathered}
\leq C \sum_{\left|\alpha_{1}\right|=m_{1},\left|\alpha_{2}\right|=m_{2}}\left(\frac{1}{|Q|} \int_{\tilde{Q}}\left|D^{\alpha_{1}} \tilde{A}_{1}(x)\right|^{p q_{1}} d x\right)^{1 / p q_{1}} \\
\left(\frac{1}{|Q|} \int_{\tilde{Q}}\left|D^{\alpha_{2}} \tilde{A}_{2}(x)\right|^{p q_{2}} d x\right)^{1 / p q_{2}} \times\left(\frac{1}{|Q|^{1-s \delta / n}} \int_{\tilde{Q}}|f(x)|_{r}^{p q_{3}} d x\right)^{1 / p q_{3}} \\
\leq C \prod_{j=1}^{2}\left(\sum_{|\alpha|=m_{j}}|| D^{\alpha} A_{j} \|_{B M O}\right) M_{\delta, s}\left(|f|_{r}\right)(\tilde{x}) .
\end{gathered}
$$

For $I_{5}$, we write

$$
\begin{aligned}
& F_{t}^{\tilde{A}}\left(h_{i}\right)(x)-F_{t}^{\tilde{A}}\left(h_{i}\right)\left(x_{0}\right) \\
& =\int_{R^{n}}\left(\frac{F_{t}(x, y)}{|x-y|^{m}}-\frac{F_{t}\left(x_{0}, y\right)}{\left|x_{0}-y\right|^{m}}\right) \prod_{j=1}^{2} R_{m_{j}}\left(\tilde{A}_{j} ; x, y\right) h_{i}(y) d y \\
& +\int_{R^{n}}\left(R_{m_{1}}\left(\tilde{A}_{1} ; x, y\right)-R_{m_{1}}\left(\tilde{A}_{1} ; x_{0}, y\right)\right) \frac{R_{m_{2}}\left(\tilde{A}_{2} ; x, y\right)}{\left|x_{0}-y\right|^{m}} F_{t}\left(x_{0}, y\right) h_{i}(y) d y \\
& +\int_{R^{n}}\left(R_{m_{2}}\left(\tilde{A}_{2} ; x, y\right)-R_{m_{2}}\left(\tilde{A}_{2} ; x_{0}, y\right)\right) \frac{R_{m_{1}}\left(\tilde{A}_{1} ; x_{0}, y\right)}{\left|x_{0}-y\right|^{m}} F_{t}\left(x_{0}, y\right) h_{i}(y) d y \\
& -\sum_{\left|\alpha_{1}\right|=m_{1}} \frac{1}{\alpha_{1} !} \int_{R^{n}}\left[\frac{R_{m_{2}}\left(\tilde{A}_{2} ; x, y\right)(x-y)^{\alpha_{1}}}{|x-y|^{m}} F_{t}(x, y)\right. \\
& \left.-\frac{R_{m_{2}}\left(\tilde{A}_{2} ; x_{0}, y\right)\left(x_{0}-y\right)^{\alpha_{1}}}{\left|x_{0}-y\right|^{m}} F_{t}\left(x_{0}, y\right)\right] \times D^{\alpha_{1}} \tilde{A}_{1}(y) h_{i}(y) d y \\
& -\sum_{\left|\alpha_{2}\right|=m_{2}} \frac{1}{\alpha_{2} !} \int_{R^{n}}\left[\frac{R_{m_{1}}\left(\tilde{A}_{1} ; x, y\right)(x-y)^{\alpha_{2}}}{|x-y|^{m}} F_{t}(x, y)\right. \\
& \left.-\frac{R_{m_{1}}\left(\tilde{A}_{1} ; x_{0}, y\right)\left(x_{0}-y\right)^{\alpha_{2}}}{\left|x_{0}-y\right|^{m}} F_{t}\left(x_{0}, y\right)\right] \times D^{\alpha_{2}} \tilde{A}_{2}(y) h_{i}(y) d y \\
& +\sum_{\left|\alpha_{1}\right|=m_{1},\left|\alpha_{2}\right|=m_{2}} \frac{1}{\alpha_{1} ! \alpha_{2} !} \int_{R^{n}}\left[\frac{(x-y)^{\alpha_{1}+\alpha_{2}}}{|x-y|^{m}} F_{t}(x, y)-\frac{\left(x_{0}-y\right)^{\alpha_{1}+\alpha_{2}}}{\left|x_{0}-y\right|^{m}} F_{t}\left(x_{0}, y\right)\right] \\
& \times D^{\alpha_{1}} \tilde{A}_{1}(y) D^{\alpha_{2}} \tilde{A}_{2}(y) h_{i}(y) d y \\
& =I_{5}^{(1)}+I_{5}^{(2)}+I_{5}^{(3)}+I_{5}^{(4)}+I_{5}^{(5)}+I_{5}^{(6)} \text {. }
\end{aligned}
$$

By Lemma 1 and the following inequality (see [19])

$$
\left|b_{Q_{1}}-b_{Q_{2}}\right| \leq C \log \left(\left|Q_{2}\right| /\left|Q_{1}\right|\right)|| b \|_{B M O} \text { for } Q_{1} \subset Q_{2},
$$


we know that, for $x \in Q$ and $y \in 2^{k+1} \tilde{Q} \backslash 2^{k} \tilde{Q}$,

$$
\begin{aligned}
\left|R_{m}(\tilde{A} ; x, y)\right| & \leq C|x-y|^{m} \sum_{|\alpha|=m}\left(\left\|D^{\alpha} A\right\|_{B M O}+\left|\left(D^{\alpha} A\right) \tilde{Q}(x, y)-\left(D^{\alpha} A\right) \tilde{Q}\right|\right) \\
& \leq C k|x-y|^{m} \sum_{|\alpha|=m}\left\|D^{\alpha} A\right\|_{B M O} .
\end{aligned}
$$

Note that $|x-y| \sim\left|x_{0}-y\right|$ for $x \in Q$ and $y \in R^{n} \backslash \tilde{Q}$, we obtain, by the conditions on $F_{t}$,

$$
\begin{aligned}
\left\|I_{5}^{(1)}\right\| & \leq C \int_{R^{n}}\left(\frac{\left|x-x_{0}\right|}{\left|x_{0}-y\right|^{m+n+1-\delta}}+\frac{\left|x-x_{0}\right|^{\varepsilon}}{\left|x_{0}-y\right|^{m+n+\varepsilon-\delta}}\right) \\
& \times \prod_{j=1}^{2} R_{m_{j}}\left(\tilde{A}_{j} ; x, y\right)|| h_{i}(y) \mid d y \\
& \leq C \prod_{j=1}^{2}\left(\sum_{|\alpha|=m_{j}}|| D^{\alpha} A_{j} \|_{B M O}\right) \\
& \times \sum_{k=0}^{\infty} \int_{2^{k+1} \tilde{Q} \backslash 2^{k} \tilde{Q}} k^{2}\left(\frac{\left|x-x_{0}\right|}{\left|x_{0}-y\right|^{n+1-\delta}}+\frac{\left|x-x_{0}\right|^{\varepsilon}}{\left|x_{0}-y\right|^{n+\varepsilon-\delta}}\right)\left|f_{i}(y)\right| d y \\
& \leq C \prod_{j=1}^{2}\left(\sum_{|\alpha|=m_{j}}|| D^{\alpha} A_{j}|| B M O\right) \\
& \times \sum_{k=1}^{\infty} k^{2}\left(2^{-k}+2^{-\varepsilon k}\right) \frac{1}{\left|2^{k} \tilde{Q}\right|^{1-\delta / n}} \int_{2^{k} \tilde{Q}}\left|f_{i}(y)\right| d y,
\end{aligned}
$$

thus, by Minkowski' inequality,

$$
\begin{aligned}
\left(\left.\sum_{i=1}^{\infty}\left\|I_{5}^{(1)}\right\|\right|^{r}\right)^{1 / r} & \leq C \prod_{j=1}^{2}\left(\sum_{|\alpha|=m_{j}}\left\|D^{\alpha} A_{j}\right\|_{B M O}\right) \\
& \times \sum_{k=1}^{\infty} k^{2}\left(2^{-k}+2^{-\varepsilon k}\right) \frac{1}{\left|2^{k} \tilde{Q}\right|^{1-\delta / n}} \int_{2^{k} \tilde{Q}}|f(y)|_{r} d y \\
& \leq C \prod_{j=1}^{2}\left(\sum_{|\alpha|=m_{j}}\left\|D^{\alpha} A_{j}\right\|_{B M O}\right) M_{\delta, 1}\left(|f|_{r}\right)(\tilde{x}) .
\end{aligned}
$$


For $I_{5}^{(2)}$, by the formula (see [4]):

$$
R_{m}(\tilde{A} ; x, y)-R_{m}\left(\tilde{A} ; x_{0}, y\right)=\sum_{|\beta|<m} \frac{1}{\beta !} R_{m-|\beta|}\left(D^{\beta} \tilde{A} ; x, x_{0}\right)(x-y)^{\beta}
$$

and Lemma 1, we have

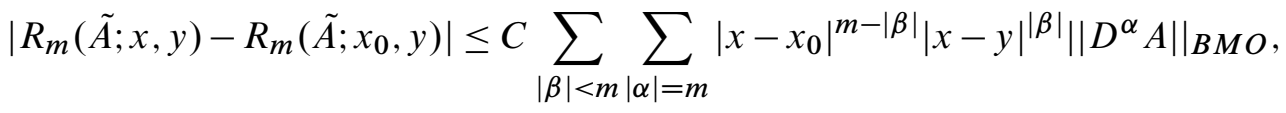

thus

$$
\begin{gathered}
\left(\sum_{i=1}^{\infty} \| I_{5}^{(2)}||^{r}\right)^{1 / r} \\
\leq C \prod_{j=1}^{2}\left(\sum_{|\alpha|=m_{j}}\left\|D^{\alpha} A_{j}\right\|_{B M O}\right) \sum_{k=0}^{\infty} \int_{2^{k+1} \tilde{Q} \backslash 2^{k} \tilde{Q}} k \frac{\left|x-x_{0}\right|}{\left|x_{0}-y\right|^{n+1-\delta}}|f(y)|_{r} d y \\
\leq C \prod_{j=1}^{2}\left(\sum_{|\alpha|=m_{j}}\left\|D^{\alpha} A_{j}\right\|_{B M O}\right) M_{\delta, 1}\left(|f|_{r}\right)(\tilde{x}) .
\end{gathered}
$$

Similarly,

$$
\left(\sum_{i=1}^{\infty}\left\|I_{5}^{(3)}\right\|^{r}\right)^{1 / r} \leq C \prod_{j=1}^{2}\left(\sum_{|\alpha|=m_{j}}\left\|D^{\alpha} A_{j}\right\|_{B M O}\right) M_{\delta, 1}\left(|f|_{r}\right)(\tilde{x}) .
$$

For $I_{5}^{(4)}$, we get

$$
\begin{aligned}
& \left(\sum_{i=1}^{\infty}\left\|I_{5}^{(4)}\right\|^{r}\right)^{1 / r} \\
& \leq C \sum_{\left|\alpha_{1}\right|=m_{1}} \int_{R^{n} \backslash \tilde{Q}}\left\|\frac{(x-y)^{\alpha_{1}} F_{t}(x, y)}{|x-y|^{m}}-\frac{\left(x_{0}-y\right)^{\alpha_{1}} F_{t}\left(x_{0}, y\right)}{\left|x_{0}-y\right|^{m}}\right\| \\
& \times\left|R_{m_{2}}\left(\tilde{A}_{2} ; x, y\right)\right|\left|D^{\alpha_{1}} \tilde{A}_{1}(y) \| h(y)\right|_{r} d y \\
& +C \sum_{\left|\alpha_{1}\right|=m_{1}} \int_{R^{n} \backslash \tilde{Q}}\left|R_{m_{2}}\left(\tilde{A}_{2} ; x, y\right)-R_{m_{2}}\left(\tilde{A}_{2} ; x_{0}, y\right)\right| \\
& \times \frac{\left|\left(x_{0}-y\right)^{\alpha_{1}}\right||| F_{t}\left(x_{0}, y\right) \|}{\left|x_{0}-y\right|^{m}}\left|D^{\alpha_{1}} \tilde{A}_{1}(y)\right||h(y)|_{r} d y \\
& \leq C \sum_{|\alpha|=m_{2}}|| D^{\alpha} A_{2} \|\left.\right|_{B M O} \sum_{\left|\alpha_{1}\right|=m_{1}} \sum_{k=1}^{\infty} k\left(2^{-k}+2^{-\varepsilon k}\right)
\end{aligned}
$$




$$
\begin{aligned}
& \times\left(\frac{1}{\left|2^{k} \tilde{Q}\right|} \int_{2^{k} \tilde{Q}}\left|D^{\alpha_{1}} \tilde{A}_{1}(y)\right|^{s^{\prime}} d y\right)^{1 / s^{\prime}}\left(\frac{1}{\left|2^{k} \tilde{Q}\right|^{1-s \delta / n}} \int_{2^{k} \tilde{Q}}|f(y)|_{r}^{s} d y\right)^{1 / s} \\
& \leq C \prod_{j=1}^{2}\left(\sum_{|\alpha|=m_{j}}|| D^{\alpha} A_{j} \|_{B M O}\right) M_{\delta, s}\left(|f|_{r}\right)(\tilde{x}) .
\end{aligned}
$$

Similarly,

$$
\left(\sum_{i=1}^{\infty}\left\|I_{5}^{(5)}\right\|^{r}\right)^{1 / r} \leq C \prod_{j=1}^{2}\left(\sum_{|\alpha|=m_{j}}\left\|D^{\alpha} A_{j}\right\|_{B M O}\right) M_{\delta, s}\left(|f|_{r}\right)(\tilde{x}) .
$$

For $I_{5}^{(6)}$, taking $q_{1}, q_{2}>1$ such that $1 / s+1 / q_{1}+1 / q_{2}=1$, then

$$
\begin{aligned}
& \left(\sum_{i=1}^{\infty} \| I_{5}^{(6)}||^{r}\right)^{1 / r} \\
& \leq C \sum_{\left|\alpha_{1}\right|=m_{1},\left|\alpha_{2}\right|=m_{2}} \int_{R^{n} \backslash \tilde{Q}}\left\|\frac{(x-y)^{\alpha_{1}+\alpha_{2}} F_{t}(x, y)}{|x-y|^{m}}-\frac{\left(x_{0}-y\right)^{\alpha_{1}+\alpha_{2}} F_{t}\left(x_{0}, y\right)}{\left|x_{0}-y\right|^{m}}\right\| \\
& \times\left|D^{\alpha_{1}} \tilde{A}_{1}(y)\right|\left|D^{\alpha_{2}} \tilde{A}_{2}(y) \| f(y)\right|_{r} d y \\
& \leq C \sum_{\left|\alpha_{1}\right|=m_{1},\left|\alpha_{2}\right|=m_{2} k=1} \sum_{k=1}^{\infty} k\left(2^{-k}+2^{-\varepsilon k}\right)\left(\frac{1}{\left|2^{k} \tilde{Q}\right|^{1-s \delta / n}} \int_{2^{k} \tilde{Q}}|f(y)|_{r}^{S} d y\right)^{1 / s} \\
& \times\left(\frac{1}{\left|2^{k} \tilde{Q}\right|} \int_{2^{k} \tilde{Q}}\left|D^{\alpha_{1}} \tilde{A}_{1}(y)\right|^{q_{1}} d y\right)^{1 / q_{1}}\left(\frac{1}{\left|2^{k} \tilde{Q}\right|} \int_{2^{k} \tilde{Q}}\left|D^{\alpha_{2}} \tilde{A}_{2}(y)\right|^{q_{2}} d y\right)^{1 / q_{2}} \\
& \leq C \prod_{j=1}^{2}\left(\sum_{|\alpha|=m_{j}}|| D^{\alpha} A_{j}\|\|_{B M O}\right) M_{\delta, s}\left(|f|_{r}\right)(\tilde{x}) .
\end{aligned}
$$

Thus

$$
I_{5} \leq C \prod_{j=1}^{2}\left(\sum_{|\alpha|=m_{j}}\left\|D^{\alpha} A_{j}\right\|_{B M O}\right) M_{\delta, s}\left(|f|_{r}\right)(\tilde{x}) .
$$

This completes the proof of Theorem 1 .

Proof of Theorem 2. We choose $1<s<p$ in Theorem 1 and by using Lemma 2, we get $\left\|T^{A}(f)\right\|_{L^{q}} \leq C\left\|\left(T^{A}(f)\right)^{\#}\right\|_{L^{q}} \leq C \prod_{j=1}^{l}\left(\sum_{|\alpha|=m_{j}}\left\|D^{\alpha} A_{j}\right\|_{B M O}\right)\left\|M_{\delta, s}\left(|f|_{r}\right)\right\|_{L^{q}}$ 


$$
\leq\left. C \prod_{j=1}^{l}\left(\sum_{|\alpha|=m_{j}}\left\|D^{\alpha} A_{j}\right\|_{B M O}\right)\|\| f\right|_{r} \|_{L^{p}}
$$

This finishes the proof.

\section{ApPLiCATIONS}

Now we give some applications of the Theorems:

Application 1. Littlewood-Paley operators.

Fixed $0 \leq \delta<n, \varepsilon>0$ and $\mu>(3 n+2-2 \delta) / n$. Let $\psi$ be a fixed function which satisfies the following properties:

(1) $\int_{R^{n}} \psi(x) d x=0$

(2) $|\psi(x)| \leq C(1+|x|)^{-(n+1-\delta)}$,

(3) $|\psi(x+y)-\psi(x)| \leq C|y|^{\varepsilon}(1+|x|)^{-(n+1+\varepsilon-\delta)}$ when $2|y|<|x|$.

We denote $\Gamma(x)=\left\{(y, t) \in R_{+}^{n+1}:|x-y|<t\right\}$ and the characteristic function of $\Gamma(x)$ by $\chi_{\Gamma(x)}$. The Littlewood-Paley multilinear operators are defined by

$$
\begin{gathered}
g_{\psi}^{A}(f)(x):=\left(\int_{0}^{\infty}\left|F_{t}^{A}(f)(x)\right|^{2} \frac{d t}{t}\right)^{1 / 2}, \\
S_{\psi}^{A}(f)(x):=\left[\iint_{\Gamma(x)}\left|F_{t}^{A}(f)(x, y)\right|^{2} \frac{d y d t}{t^{n+1}}\right]^{1 / 2}
\end{gathered}
$$

and

$$
g_{\mu}^{A}(f)(x):=\left[\iint_{R_{+}^{n+1}}\left(\frac{t}{t+|x-y|}\right)^{n \mu}\left|F_{t}^{A}(f)(x, y)\right|^{2} \frac{d y d t}{t^{n+1}}\right]^{1 / 2},
$$

where

$$
\begin{gathered}
F_{t}^{A}(f)(x):=\int_{R^{n}} \frac{\prod_{j=1}^{l} R_{m_{j}+1}\left(A_{j} ; x, y\right)}{|x-y|^{m}} \psi_{t}(x-y) f(y) d y, \\
F_{t}^{A}(f)(x, y):=\int_{R^{n}} \frac{\prod_{j=1}^{l} R_{m_{j}+1}\left(A_{j} ; x, z\right)}{|x-z|^{m}} f(z) \psi_{t}(y-z) d z
\end{gathered}
$$

and $\psi_{t}(x):=t^{-n+\delta} \psi(x / t)$ for $t>0$. Set $F_{t}(f)(y)=f * \psi_{t}(y)$. We also define

and

$$
\begin{gathered}
g_{\psi}(f)(x):=\left(\int_{0}^{\infty}\left|F_{t}(f)(x)\right|^{2} \frac{d t}{t}\right)^{1 / 2}, \\
S_{\psi}(f)(x):=\left(\iint_{\Gamma(x)}\left|F_{t}(f)(y)\right|^{2} \frac{d y d t}{t^{n+1}}\right)^{1 / 2}
\end{gathered}
$$

$$
g_{\mu}(f)(x):=\left(\iint_{R_{+}^{n+1}}\left(\frac{t}{t+|x-y|}\right)^{n \mu}\left|F_{t}(f)(y)\right|^{2} \frac{d y d t}{t^{n+1}}\right)^{1 / 2},
$$


which are the Littlewood-Paley operators (see[20]). Let $H$ be the space

$$
H:=\left\{h:\|h\|=\left(\int_{0}^{\infty}|h(t)|^{2} d t / t\right)^{1 / 2}<\infty\right\}
$$

or

$$
H:=\left\{h:\|h\|=\left(\iint_{R_{+}^{n+1}}|h(y, t)|^{2} d y d t / t^{n+1}\right)^{1 / 2}<\infty\right\},
$$

then, for each fixed $x \in R^{n}, F_{t}^{A}(f)(x)$ and $F_{t}^{A}(f)(x, y)$ may be viewed as the mapping from $[0,+\infty)$ to $H$, and it is clear that

$$
\begin{gathered}
g_{\psi}^{A}(f)(x)=\left\|F_{t}^{A}(f)(x)\right\|, \quad g_{\psi}(f)(x)=\left\|F_{t}(f)(x)\right\|, \\
S_{\psi}^{A}(f)(x)=\left\|\chi_{\Gamma(x)} F_{t}^{A}(f)(x, y)\right\|, \quad S_{\psi}(f)(x)=\left\|\chi_{\Gamma(x)} F_{t}(f)(y)\right\|
\end{gathered}
$$

and

$$
\begin{gathered}
g_{\mu}^{A}(f)(x)=\left\|\left(\frac{t}{t+|x-y|}\right)^{n \mu / 2} F_{t}^{A}(f)(x, y)\right\|, \\
g_{\mu}(f)(x)=\left\|\left(\frac{t}{t+|x-y|}\right)^{n \mu / 2} F_{t}(f)(y)\right\| .
\end{gathered}
$$

It is easily to see that $g_{\psi}, S_{\psi}$ and $g_{\mu}$ satisfy the conditions of Theorems 1 and 2 (see [9-11]), thus Theorems 1 and 2 hold for $g_{\psi}^{A}, S_{\psi}^{A}$ and $g_{\mu}^{A}$.

Application 2. Marcinkiewicz operators.

Fixed $0 \leq \delta<n$, Fix $\lambda>\max (1,2 n /(n+2-2 \delta))$ and $0<\gamma \leq 1$. Let $\Omega$ be homogeneous of degree zero on $R^{n}$ with $\int_{S^{n-1}} \Omega\left(x^{\prime}\right) d \sigma\left(x^{\prime}\right)=0$. Assume that $\Omega \in$ Li $p_{\gamma}\left(S^{n-1}\right)$. The Marcinkiewicz multilinear operators are defined by

$$
\begin{gathered}
\mu_{\Omega}^{A}(f)(x):=\left(\int_{0}^{\infty}\left|F_{t}^{A}(f)(x)\right|^{2} \frac{d t}{t^{3}}\right)^{1 / 2} \\
\mu_{S}^{A}(f)(x):=\left[\iint_{\Gamma(x)}\left|F_{t}^{A}(f)(x, y)\right|^{2} \frac{d y d t}{t^{n+3}}\right]^{1 / 2}
\end{gathered}
$$

and

$$
\mu_{\lambda}^{A}(f)(x):=\left[\iint_{R_{+}^{n+1}}\left(\frac{t}{t+|x-y|}\right)^{n \lambda}\left|F_{t}^{A}(f)(x, y)\right|^{2} \frac{d y d t}{t^{n+3}}\right]^{1 / 2},
$$

where

$$
F_{t}^{A}(f)(x):=\int_{|x-y| \leq t} \frac{\prod_{j=1}^{l} R_{m_{j}+1}\left(A_{j} ; x, y\right)}{|x-y|^{m}} \frac{\Omega(x-y)}{|x-y|^{n-1-\delta}} f(y) d y
$$


and

$$
F_{t}^{A}(f)(x, y):=\int_{|y-z| \leq t} \frac{\prod_{j=1}^{l} R_{m_{j}+1}\left(A_{j} ; y, z\right)}{|y-z|^{m}} \frac{\Omega(y-z)}{|y-z|^{n-\delta-1}} f(z) d z .
$$

Set

$$
F_{t}(f)(x):=\int_{|x-y| \leq t} \frac{\Omega(x-y)}{|x-y|^{n-1-\delta}} f(y) d y .
$$

We also define

$$
\begin{gathered}
\mu_{\Omega}(f)(x):=\left(\int_{0}^{\infty}\left|F_{t}(f)(x)\right|^{2} \frac{d t}{t^{3}}\right)^{1 / 2} \\
\mu_{S}(f)(x):=\left(\iint_{\Gamma(x)}\left|F_{t}(f)(y)\right|^{2} \frac{d y d t}{t^{n+3}}\right)^{1 / 2}
\end{gathered}
$$

and

$$
\mu_{\lambda}(f)(x):=\left(\iint_{R_{+}^{n+1}}\left(\frac{t}{t+|x-y|}\right)^{n \lambda}\left|F_{t}(f)(y)\right|^{2} \frac{d y d t}{t^{n+3}}\right)^{1 / 2},
$$

which are the Marcinkiewicz operators (see[21]). Let $H$ be the space

$$
H:=\left\{h:\|h\|=\left(\int_{0}^{\infty}|h(t)|^{2} d t / t^{3}\right)^{1 / 2}<\infty\right\}
$$

or

$$
H:=\left\{h:\|h\|=\left(\iint_{R_{+}^{n+1}}|h(y, t)|^{2} d y d t / t^{n+3}\right)^{1 / 2}<\infty\right\} .
$$

Then, it is clear that

$$
\begin{gathered}
\mu_{\Omega}^{A}(f)(x)=\left\|F_{t}^{A}(f)(x)\right\|, \quad \mu_{\Omega}(f)(x)=\left\|F_{t}(f)(x)\right\| \\
\mu_{S}^{A}(f)(x)=\left\|\chi_{\Gamma(x)} F_{t}^{A}(f)(x, y)\right\|, \quad \mu_{S}(f)(x)=\left\|\chi_{\Gamma(x)} F_{t}(f)(y)\right\|
\end{gathered}
$$

and

$$
\begin{gathered}
\mu_{\lambda}^{A}(f)(x)=\left\|\left(\frac{t}{t+|x-y|}\right)^{n \lambda / 2} F_{t}^{A}(f)(x, y)\right\|, \\
\mu_{\lambda}(f)(x)=\left\|\left(\frac{t}{t+|x-y|}\right)^{n \lambda / 2} F_{t}(f)(y)\right\| .
\end{gathered}
$$

It is easily to see that $\mu_{\Omega}, \mu_{S}$ and $\mu_{\lambda}$ satisfy the conditions of Theorems 1 and 2 (see $[12,21])$, thus Theorems 1 and 2 hold for $\mu_{\Omega}^{A}, \mu_{S}^{A}$ and $\mu_{\lambda}^{A}$.

Application 3. Bochner-Riesz operator . 
Let $\delta>(n-1) / 2, B_{t}^{\delta}\left(f \hat{)(\xi)}=\left(1-t^{2}|\xi|^{2}\right)_{+}^{\delta} \hat{f}(\xi)\right.$ and $B_{t}^{\delta}(z)=t^{-n} B^{\delta}(z / t)$ for $t>0$. Set

$$
F_{\delta, t}^{A}(f)(x):=\int_{R^{n}} \frac{\prod_{j=1}^{l} R_{m_{j}+1}\left(A_{j} ; x, y\right)}{|x-y|^{m}} B_{t}^{\delta}(x-y) f(y) d y,
$$

The maximal Bochner-Riesz multilinear operator are defined by

$$
B_{\delta, *}^{A}(f)(x):=\sup _{t>0}\left|B_{\delta, t}^{A}(f)(x)\right| .
$$

We also define

$$
B_{\delta, *}(f)(x):=\sup _{t>0}\left|B_{t}^{\delta}(f)(x)\right|,
$$

which is the maximal Bochner-Riesz operator (see [13]). Let $H$ be the space $H:=$ $\left\{h:|| h||=\sup _{t>0}|h(t)|<\infty\right\}$, then

$$
B_{\delta, *}^{A}(f)(x)=\left\|B_{\delta, t}^{A}(f)(x)\right\|, \quad B_{*}^{\delta}(f)(x)=\left\|B_{t}^{\delta}(f)(x)\right\| .
$$

It is easily to see that $B_{\delta, *}^{A}$ satisfies the conditions of Theorems 1 and 2 (see [22]), thus Theorems 1 and 2 hold for $B_{\delta, *}^{A}$.

\section{REFERENCES}

[1] S. Chanillo, "A note on commutators," Indiana Univ. Math. J., vol. 31, pp. 7-56, 1982.

[2] J. Cohen and J. Gosselin, "On multilinear singular integral operators on $R^{n}$," Studia Math., vol. 72, no. 2, pp. 199-223, 1982.

[3] J. Cohen, "A sharp estimate for a multilinear singular integral in $\mathbb{R}^{n}$," Indiana Univ. Math. J., vol. 30, pp. 693-702, 1981.

[4] J. Cohen and J. Gosselin, "A BMO estimate for multilinear singular integrals," Ill. J. Math., vol. 30, pp. 445-464, 1986.

[5] Y. Ding and S. Z. Lu, "Weighted boundedness for a class of rough multilinear operators," Acta Math. Sin., Engl. Ser., vol. 17, no. 3, pp. 517-526, 2001.

[6] C. Fefferman and E. M. Stein, "Some maximal inequalities," Am. J. Math., vol. 93, pp. 107-115, 1971.

[7] J. García-Cuerva and J. L. Rubio de Francia, Weighted norm inequalities and related topics, ser. North-Holland Mathematics Studies. Amsterdam-New York-Oxford: North-Holland, 1985, vol. 116.

[8] G. Hu and D. Yang, "A variant sharp estimate for multilinear singular integral operators," Stud. Math., vol. 141, no. 1, pp. 25-42, 2000.

[9] L. Liu, "Weighted weak type estimates for commutators of Littlewood-Paley operator," Jap. J. Math., New Ser., vol. 29, no. 1, pp. 1-13, 2003.

[10] L. Liu, "A sharp endpoint estimate for a multilinear Littlewood-Paley operator," Georgian Math. J., vol. 11, no. 2, pp. 361-370, 2004.

[11] L. Liu, "Sharp endpoint inequality for multilinear Littlewood-Paley operator," Kodai Math. J., vol. 27, no. 2, pp. 134-143, 2004.

[12] L. Liu, "A sharp estimate for multilinear Marcinkiewicz integral operator," Asian J. Math., vol. 9, no. 2, pp. 177-184, 2005.

[13] S. Lu, Four lectures on real $H^{p}$ spaces. Singapore: World Scientific, 1995. 
[14] Y. Meyer and R. Coifman, Wavelets: Calderón-Zygmund and multilinear operators, ser. Cambridge Studies in Advanced Mathematics. Cambridge: Cambridge University Press, 1997, vol. 48.

[15] C. Pérez and R. Trujillo-González, "Sharp weighted estimates for multilinear commutators," $J$. Lond. Math. Soc., II. Ser., vol. 65, no. 3, pp. 672-692, 2002.

[16] C. Pérez, "Endpoint estimates for commutators of singular integral operators," J. Funct. Anal., vol. 128, no. 1, pp. 163-185, 1995.

[17] C. Pérez and G. Pradolini, "Sharp weighted endpoint estimates for commutators of singular integrals," Mich. Math. J., vol. 49, no. 1, pp. 23-37, 2001.

[18] C. Pérez and R. Trujillo-González, "Sharp weighted estimates for vector-valued singular integral operators and commutators," Tohoku Math. J., II. Ser., vol. 55, no. 1, pp. 109-129, 2003.

[19] E. M. Stein, Harmonic analysis: Real-variable methods, orthogonality, and oscillatory integrals. With the assistance of Timothy S. Murphy, ser. Princeton Mathematical Series. Princeton, NJ: Princeton University Press, 1993, vol. 43.

[20] A. Torchinsky, Real-variable methods in harmonic analysis, ser. Pure and Applied Mathematics. Orlando: Academic Press, Inc. (Harcourt Brace Jovanovich, Publishers), 1986, vol. 123.

[21] A. Torchinsky and S. Wang, "A note on the Marcinkiewicz integral," Colloq. Math., vol. 60/61, no. 1, pp. 235-243, 1990.

[22] B. S. Wu and L. Z. Liu, "A sharp estimate for multilinear Bochner-Riesz operator," Stud. Sci. Math. Hung., vol. 42, no. 1, pp. 47-59, 2005.

Author's address

Xiaosha Zhou

College of Mathematics, Changsha University of Science and Technology, Changsha 410077, P. R. of China

E-mail address: zhouxiaosha57@126.com 